\title{
OPTIMAL BOUNDS FOR TOADER MEAN IN TERMS OF ARITHMETIC AND CONTRAHARMONIC MEANS
}

\section{Ying-Qing Song, WeI-Dong JiAng, Yu-Ming Chu And DAn-DAn Yan}

Abstract. We find the greatest value $\alpha_{1}$ and $\alpha_{2}$, and the least values $\beta_{1}$ and $\beta_{2}$, such that the double inequalities $\alpha_{1} C(a, b)+\left(1-\alpha_{1}\right) A(a, b)<T(a, b)<\beta_{1} C(a, b)+\left(1-\beta_{1}\right) A(a, b)$ and $\alpha_{2} / A(a, b)+\left(1-\alpha_{2}\right) / C(a, b)<1 / T(a, b)<\beta_{2} / A(a, b)+\left(1-\beta_{2}\right) / C(a, b)$ hold for all $a, b>0$ with $a \neq b$. As applications, we get new bounds for the complete elliptic integral of the second kind. Here, $C(a, b)=\left(a^{2}+b^{2}\right) /(a+b), A(a, b)=(a+b) / 2$, and

$$
T(a, b)=\frac{2}{\pi} \int_{0}^{\pi / 2} \sqrt{a^{2} \cos ^{2} \theta+b^{2} \sin ^{2} \theta} d \theta
$$

denote the contraharmonic, arithmetic, and Toader means of two positive numbers $a$ and $b$, respectively.

Mathematics subject classification (2010): 26E60, 33E05.

Keywords and phrases: Contraharmonic mean, arithmetic mean, Toader mean, complete elliptic integrals.

\section{REFERENCES}

[1] GH. ToAder, Some mean values related to the arithmetic-geometric mean, J. Math. Anal. Appl., 1998, 218 (2): 358-368.

[2] E. Neuman, Bounds for symmetric elliptic integrals, J. Approx. Theory, 2003, 122 (2): 249-259.

[3] H. Kazi And E. Neuman, Inequalities and bounds for elliptic integrals, J. Approx. Theory, 2007, 146 (2): 212-226.

[4] H. KaZI AND E. Neuman, Inequalities and bounds for elliptic integrals II, Special functions and orthogonal Polynomials, 127-138, Contemp. Math., 471, Amer. Math. Soc., Providence, RI, 2008.

[5] M.-K. WANG, Y.-M. CHU, S.-L. QIU AND Y.-P. JIANG, Bounds for the perimeter of an ellipse, J. Approx. Theory, 2012, 164 (7): 928-937.

[6] Y.-M. ChU And M.-K. WAng, Optimal Lehmer mean bounds for the Toader mean, Results Math., 2012, 61 (3-4): 223-229.

[7] Y.-M. ChU, M.-K. WANG AND S.-L. QIU, Optimal combinations bounds of root-square and arithmetic means for Toader mean, Proc. Indian Acad. Sci. Math. Sci., 2012, 122 (1): 41-51.

[8] Y.-M. CHU AND M.-K. WANG, Inequalities between arithmetic-geometric, Gini, and Toader means, Abstr. Appl. Anal., 2012, Article ID 830585, 11 pages.

[9] Y.-M. CHU, M.-K. WANG, S.-L. QIU AND Y.-F. QIU, Sharp generalized Seiffert mean bounds for Toader mean, Abstr. Appl. Anal., 2011, Article ID 605259, 8 pages.

[10] M. VuORINEN, Hypergeometric functions in geometric function theory, Special functions and differential equations (Madras, 1997), 119-126, Allied Publ., New Delhi, 1998.

[11] S.-L. QIU AND J.-M. SHEN, On two problems concerning means, Journal of Hangzhou Insitute of Electronic Engineering, 1997, 17 (3): 1-7 (in Chinese).

[12] R. W. BARNARD, K. PEARCE AND K. C. RICHARDS, An inequality involving the generalized hypergeometric function and the arc length of an ellipse, SIAM J. Math. Anal., 2000, 31 (3): 693-699.

[13] H. AlZER AND S.-L. QIU, Monotonicity theorems and inequalities for the complete elliptic integrals, J. Comput. Appl. Math., 2004, 172 (2): 289-312. 
[14] G. D. Anderson, M. K. Vamanamurthy and M. Vuorinen, Conformal Invariants, Inequalities, and Quasiconformal Maps, John Wiley \& Sons, New York, 1997. 